Bustos, E. H. (2015). Tendencias investigativas en territorio y su importancia en la formación de profesionales. Revista Lebret, 7. Bucaramanga, Colombia: Universidad Santo Tomás, pp. 261 - 279. ISSN: 2145-5996.

\title{
Tendencias investigativas en territorio y su importancia en la formación de profesionales"
}

\author{
Investigative trends in territory and its importance in the \\ formation of professionals
}

Edier Hernan Bustos Velazco

\begin{abstract}
Resumen
El artículo establece desde los antecedentes cuatro tendencias: (a) territorio como campo de poder jurídico y político, (b) territorio como ambiente, c) territorio como construcción social y (d) territorio como espacio físico. En cada una de ellas se presentan de forma sintética y concisa las posturas de investigadores, lo que hace de esta publicación un insumo para todas aquellas personas que por su acción profesional o por curiosidad investigativa deseen desarrollar investigaciones relacionadas con el territorio como campo de estudio.
\end{abstract}

\section{Palabras clave}

Territorio, territorialidad, ciencias de la tierra.

\section{Códigos de clasificación JEL: I38, J11, K32, 015, Q15,Q20}

\begin{abstract}
This essay derives four trends from previous research: (a) territory as the field of legal and political power, (b) territory as environment, (c) territory as a social construct, and (d) territory as physical space. In each trend, the positions of researchers is summarized, making this publication a source for all those who, driven by professional interest or investigative curiosity, wish to do research related to the territory as a field of study.
\end{abstract}

\section{Keywords}

Territory, territoriality, earth sciences.

* $\quad$ El artículo surge de la investigación doctoral "Concepciones de territorio de docentes universitarios formadores de profesionales de las ciencias de la tierra: estudio comparado en dos universidades públicas ubicadas en contextos culturalmente diferenciados", avalada por el Centro de Investigación y Desarrollo Científico de la Universidad Distrital Francisco José de Caldas.

1 Estudiante del Doctorado Interinstitucional en Educación (DIE), énfasis Enseñanza de las Ciencias Contexto y Diversidad Cultural. Estudiante del Doctorado en Geografía. Docente Universidad Distrital Francisco José de Caldas. Correo electrónico: ehbustosv@udistrital.edu.co 


\section{Metodología}

La investigación tiene como uno de sus fines, configurar categorías de análisis alrededor de la idea de territorio, para lograrlo, se indagaron diversos motores de búsqueda, luego de ello, se mapearon los contenidos conceptuales relevantes, proceso metodológico que se desarrolló en cuatro fases: a) búsqueda de artículos en bases especializadas relacionados con las concepciones de territorio, b) organización de la información en matriz Excel, c) mediante el análisis de contenidos determinar los referentes, d) las herramienta de filtros de Excel y tablas dinámicas permitió caracterizar las categorías de análisis.

Tabla 1. Ficha bibliográfica

\begin{tabular}{|c|c|c|c|c|c|c|}
\hline $\mathrm{N}^{\circ}$ & Año & País & $\begin{array}{c}\text { Palabras } \\
\text { clave }\end{array}$ & Resumen & Metodología & $\begin{array}{c}\text { Campo } \\
\text { Temático }\end{array}$ \\
\hline $\begin{array}{c}\text { Orden de la } \\
\text { publicación }\end{array}$ & $\begin{array}{c}\text { Fecha de } \\
\text { publicación }\end{array}$ & $\begin{array}{c}\text { Lugar de la } \\
\text { publicación }\end{array}$ & $\begin{array}{c}\text { Términos que } \\
\text { identifican el } \\
\text { contenido }\end{array}$ & $\begin{array}{c}\text { Referido al } \\
\text { documento }\end{array}$ & $\begin{array}{c}\text { Enfoque } \\
\text { metodológico }\end{array}$ & $\begin{array}{c}\text { Contenido } \\
\text { temático de la } \\
\text { publicación }\end{array}$ \\
\hline
\end{tabular}

Fuente: autor.

Con este método de estudio de información se seleccionaron cuatro grandes categorías de análisis: territorio desde el campo jurídico, territorio desde el campo ambiental, territorio como construcción social y territorio como espacio físico.

\section{Territorio como campo de poder jurídico}

Al hacer referencia al territorio como campo de poder jurídico y político, se pudo inferir que está directamente relacionado con todas las actividades de la sociedad, donde emergen tensiones entre los diferentes actores que en él interactúan. Aquí las luchas desde la legalidad pueden ser mediadas por las reglas aceptadas por la sociedad; desde esta perspectiva, el campo jurídico es susceptible a una variedad de interpretaciones, que son mediadas desde un aspecto jurídico, definido por unas reglas, que a la luz de la reglamentación constitucional validan lo que es y no es aceptado por la sociedad. En fin, entrar en el campo jurídico, implica vigilar las tensiones entre los diferentes actores de la sociedad que alrededor de la posesión del territorio, demandan sus derechos, llámense estos actores; indígenas, afrodescendientes, comunidades rom, empresarios, raizales, ganaderos, multinacionales, campesinos, entre otros, que sin una jurisprudencia adecuada no alcanzarían a validar lo que uno $\mathrm{u}$ otro considera su verdad.

Por las dinámicas de investigación, se incluyeron en esta dimensión jurídica del territorio, aquellas investigaciones que específicamente en Colombia han indagado en las cosmovisiones y derechos territoriales para las comunidades indígenas y afrodescendientes, sustentadas a la luz de la Constitución Política de 1991, el reconocimiento a la multiculturalidad y diversidad cultural. En Colombia las luchas 
de las comunidades ancestrales han estado en continuas y permanentes tensiones, ligadas con el reconocimiento como pueblos con autonomía territorial, esto se ve reflejado desde el ámbito político en tres escenarios:

el transnacional, el nacional y el local. Sin embargo, paralelamente en estos tres escenarios se relacionan procesos específicos en torno a recursos naturales y control territorial, lo que conlleva al análisis de la ecogubernamentalidad transnacional; el reconocimiento-desconocimiento de las ciudadanías nacionales plurales; y la imposición local de soberanías de hecho (Ulloa, 2010, p. 76).

Escenarios que para el caso colombiano han estado disputados por diferentes actores a lo largo de su historia, buscando explotar los recursos naturales y minerales. Esta es una de las razones más poderosas que tienen las comunidades para ampararse en la legislación y obligar a todos aquellos actores externos, a que antes de efectuar cualquier proyecto en sus territorios, deban realizar la Consulta Previa $^{2}$ como proceso de concertación.

Con la Ley 70 de 1993, se configuró la posibilidad de establecer los Consejos Comunitarios para las comunidades negras, lo que les permitió realizar títulos de manera colectiva a los territorios ancestralmente ocupados, sin embargo:

...los logros de la Ley 70 de 1993 son muy limitados, a pesar de que reconociera la titularidad de derechos a la población afrodescendiente, como la identidad, la participación, el territorio, entre otros, sus impactos no han sido lo suficientemente amplios, teniendo en cuenta la situación de desprotección de la cual aún es víctima hoy la gran mayoría de la población afrodescendiente. Dentro de los logros de la normativa se destacan algunos avances en el campo de la etnoeducación y la titulación de cerca de seis millones de hectáreas en el Pacífico colombiano (Coronado, 2006, p. 60).

Por lo expuesto, pareciera que la problemática de tierras para las comunidades ancestrales está resuelta, pero la mayor parte del territorio reconocido es de baja producción; lo que ha llevado a la pobreza extrema de las comunidades ubicadas en resguardos, a pesar de que la Asamblea Nacional Constituyente haya determinado que:

...Colombia ratificará, en directa conexión con las disposiciones constitucionales, la convención 169 de 1989 de la OIT sobre pueblos indígenas y tribales y la tradujera en derecho nacional mediante la ley 21 de 1991 (...) Según la Corte Constitucional, las disposiciones constitucionales y las regulaciones de la convención 169 de la OIT forman un bloque de constitucionalidad (Semper, 2006, p. 763).

La Constitución articulada al Convenio 169 y a la consulta previa, ha procurado velar por la defensa y reconocimiento de los territorios ancestrales, entre los que se

2 La figura de la Consulta Previa, pareciera ser uno de los elementos más utilizados por los grupos étnicos y defendido por la Corte Constitucional en pro del derecho que tienen las comunidades ancestrales a habitar sus territorios y para ello se ha utilizado el concepto de tutela. 
encuentran los resguardos ${ }^{3}$ indígenas que garantizan el fortalecimiento cultural y la identidad de las comunidades, lo que hace de ese territorio, "un referente principal de su identidad, una cuestión construida históricamente más que ontológica" (Boiser, 2007 , p. 19) donde por las circunstancias socio territoriales, las comunidades han tenido diferentes momentos históricos relativamente recientes para su reconocimiento;

Los pueblos indígenas fueron reconocidos en el Convenio 169 de la OIT de 1989, ratificado por Colombia en la Ley 21 del 4 marzo de 1991 (Convenio 169 OIT, 1992); las comunidades negras fueron reconocidas en 1993 con la Ley 70, reglamentaria del artículo transitorio 55 de la Constitución Política Nacional. En cuanto al pueblo rom (gitano) el Ministerio del Interior, a través de la Dirección General de Asuntos Indígenas, lo reconoció como grupo étnico solo a partir del 20 de febrero de 1998. En 1999 fue aprobada legalmente por Resolución 022 del 2 de septiembre del mismo año, la organización Proceso Organizativo del Pueblo Rom de Colombia (Cepal, 2005, p. 136).

No obstante, y a pesar de la legislación como se ha citado; historicamente se han seguido vulnerando los derechos de las comunidades ancestrales. Parafraceando a Mançano (2008), para el de las comunidades indígenas de la sierra nevada de Santa Martha, emergen disputas territoriales motivadas por intereses económicos abordados desde la explotación de los recursos naturales y minerales. Estas disputas territoriales se desarrollan por intereses particulares, en el ámbito político, teórico e ideológico, lo que nos conduce a reflexionar es que a pesar de que las comunidades tienen derecho a su territorio, este es relativo y está supeditado a los intereses de actores externos a sus territorios que desean como cual ave rapaz, apoderarse de las riquezas que en él subyacen.

Por lo anterior, no basta con reconocer la relación que tienen las comunidades ancestrales con sus territorios, el Estado debe ir más allá, brindando una protección efectiva a las comunidades, ya que sin amparo continuaran aumentando los niveles de desplazamiento forzado. Por lo tanto, “...tratar la problemática de los territorios de conflicto en Colombia implica abordar el tema del desplazamiento forzado, bien desde condiciones sociopolíticas, bien desde condiciones económicas" (Nates y Raymond, 2006, p. 99).

Para hacer un recuento, el reconocimiento de tierras, para el caso colombiano, radica en la Constitución Política colombiana de $1991^{4}$, en la que se da reconocimiento

3 "El artículo 1502 del Código Civil señala en su tercer numeral que un requisito legal para que cualquier acuerdo de voluntades de carácter particular - un contrato de compraventa, de arrendamiento, etc. sea jurídicamente válido es que recaiga sobre un objeto lícito, es decir, un bien que sea lícito enajenar. Dentro del sistema jurídico colombiano, existen muchos bienes cuya enajenación está prohibida. Se les denomina bienes inalienables. El típico ejemplo es el de los resguardos de las comunidades indígenas y los territorios colectivos de las negritudes (...) de acuerdo con la Constitución Política y las leyes nacionales, los resguardos y los territorios colectivos son bienes inalienables, imprescriptibles e inembargables" (Sánchez, et al. 2009, p. 46).

4 Disposiciones que definen el contenido del derecho fundamental al territorio de las comunidades negras, entre ellas: - Protección de lenguas y dialectos de grupos étnicos (artículo 10).

- Imprescriptibilidad, inembargabilidad e inalienabilidad de los territorios colectivos de las comunidades étnicas (artículo 329).

- Derecho de consulta previa para la explotación de recursos naturales sobre los territorios colectivos (artículo 329)

- Autonomía de las formas de gobierno, planeación y organización social (artículo 330). 
a las comunidades indígenas y afrocolombianas, por lo que se instauró desde ese momento histórico una realidad jurídica, que debe conocer toda la sociedad y en especial aquellos profesionales de las ciencias de la tierra, que son educadores y que por acción profesional están involucrados en proyectos ubicados en territorios ancestrales. Lo que invita a conocer a profundidad la normatividad, dadas las implicaciones que tienen las dinámicas políticas y sociales en el contexto de las comunidades, quienes se han visto abocadas al uso del "campo jurídico"s, que en su normatividad aborda: la Constitución Política de $1991^{6}$, el Convenio de la OIT sobre Pueblos Indígenas y Tribales que entra en vigor el 4 de enero de 19697, la Ley 70 de $1993^{8}$ y la Consulta Previa; herramienta que desde lo jurídico, utilizan las comunidades para defender el derecho a su territorio; en este sentido vale la pena resaltar el huso que hacen de la Constitución Política; especialmente a los artículos $5,7,10,13,329$ y 330 .

\section{Territorio como campo ambiental}

\section{En este documento asumimos como ambiente}

un sistema dinámico definido por las interacciones físicas, biológicas, sociales y culturales percibidas o no entre los seres humanos y los demás seres vivientes y todos los elementos del medio donde se desenvuelven, sean elementos naturales o transformados por el hombre (MADS ${ }^{9}$ 2012, p. 27)

Lejos de las visiones de desarrollo sostenible ${ }^{10}$, vemos el territorio con los ojos de un desarrollo sustentable, dado que es aquí en el territorio latinoamericano justamente donde

La sustentabilidad se enraiza en bases ecológicas e identidades culturales... a nivel local es donde se forjan las identidades culturales, donde estas se expresan como una valorización social de los recursos económicos y como estrategias para la reapropiación de la naturaleza (Leff, 2006, p. 269).

Por lo expuesto, corresponden a esta dimensión aquellas investigaciones como la de Moreno (2010) que relacionan el territorio con los sistemas ambientales, en las que los juicios de valor los da la sociedad, se dan como una relación de cambio o como

5 "Entendiendo campo en términos de Bourdieu como un espacio específico en donde suceden una serie de interacciones; por otra parte lo define como un sistema particular de relaciones objetivas que pueden ser de alianza o conflicto, de concurrencia o cooperación entre posiciones diferentes, socialmente definidas e instituidas independientes de la existencia física y de los agentes que la ocupan" (Moreno, 2006, p. 16).

6 http://www.contraloriapereira.gov.co/files.php?fid=24\&fcid=2\&PHPSESSID=0af317a9c52c6247711881ae5e8d5551 www.cdi.gob.mx/transparencia/convenio169_oit.pdf

http://www.alcaldiabogota.gov.co/sisjur/normas/Norma1.jsp?i=7388

Ministerio de Ambiente y Desarrollo Sostenible. Educación Ambiental. Política Nacional.

10 ...el discurso del crecimiento sostenible levanta una cortina de humo que vela "La geopolítica de la biodiversidad y del desarrollo sustentable no solo prolonga e intensifica los anteriores procesos de apropiación destructiva de los recursos naturales, sino que cambia las formas de intervención y apropiación de la naturaleza y lleva a su límite la lógica de la racionalidad económica... las causas reales de la crisis ecológica. Así, ante el calentamiento global del planeta, se desconoce la degradación entrópica que produce la actividad económica ejercida bajo la racionalidad económica (cuyo último grado de degradación es el calor) y se niega el origen antropogénico del fenómeno al calificar sus efectos como desastres "naturales" (Leff, 2006, p. 265). 
una significación cultural, en tal sentido y apoyados en el trabajo de Milton Santos (2000) se puede afirmar que el conocimiento del territorio y de las relaciones del hombre con diferentes ambientes de fauna y flora, es el resultado de una compleja y dinámica interacción que está condicionada por factores culturales y ambientales; parafraseando a (Geertz, 1992, p. 20), en la cultura se encuentran representados todos los esquemas que históricamente se han transmitido de generación en generación, lo que permite preservar la identidad y los conocimientos ancestrales. Por su parte, Sauve (2003), al referirse al medio ambiente y su relación con el territorio, establece que el medio ambiente se entiende como: "a) el territorio (lugar de pertenencia y de identidad cultural), b) el paisaje (por recorrer, por interpretar)" (p. 4). En su propuesta destaca la idea de realizar proyectos comunitarios, donde el ecodesarrollo comunitario local o regional es entendido como la defensa de los parques y zonas protegidas. Irastorza (2006) coincide con Sauve (2003) al proponer que es indispensable estudiar las interacciones temporales y espaciales entre paisaje, flora, fauna y relaciones culturales, así, la idea de territorio es concebida "como el lugar de identidad de una naturaleza cultural que tiene como objetivo, reconocer el estrecho lazo entre naturaleza y la cultura, lo cual permite clarificar su propia cosmología, valorizar la dimensión cultural de su relación con el ambiente" (Sauve, 2003 , p. 9), por lo que equiparar el territorio como ambiente implica reconocer la relación dinámica que existe entre el ser humano, su cultura, la vida animal y vegetal.

En Colombia, durante su asentamiento y construcción de poblados, las riberas de los ríos, ciénagas y lagos han sido y fueron elemento fundamental para su subsistencia y desarrollo, "históricamente se ha asumido la relación territorial entre humedales, ríos, ciudades y paisaje" (Escobar, 2001, p. 148), ha afectado los ecosistemas naturales, lo que genera el desplazamiento de la fauna, por la contaminación del agua con desechos orgánicos e industriales. Sin embargo, repensar el territorio colombiano con la idea de sustentabilidad, invita a asumir posturas críticas frente a la protección del medio ambiente, lo que requiere de una muy buena gestión ambiental, visto como un " proceso coherente de planeación, ejecución y seguimiento de políticas y acciones ambientales" (Osorio, 2001, p. 132), orientado a mejorar la calidad del territorio urbano o rural al proponer y construir formas alternativas de recursos económicos para las comunidades relacionadas con el agua y su entorno ambiental.

Para Otero (2006), el estudio del territorio tiene importancia en la medida que sea competente, específicamente de los destinos turísticos al posibilitar la interacción del hombre con la naturaleza. Desde esta perspectiva, el nuevo entorno adquiere otros intereses, como la capacidad de vincular las culturas en las nuevas dinámicas sociales y económicas. Indudablemente, cada proceso de transformación social deriva de las relaciones de la sociedad en sí misma, con la fauna y la flora, prácticas culturales que en una sociedad permiten articular el proyecto de sustentabilidad y por ello la importancia del territorio local reside en la relación de los sujetos con su entorno, lo que resulta clave "por tanto la racionalidad ambiental implica, necesariamente, 
el recorrido de sendas alternativas, de vías ideológicas diferentes para establecer nuevos vínculos entre los distintos sistemas culturales y los sistemas naturales" (March, 2005, p. 16), que aseguran que un desarrollo sustentable responsable con las comunidades debe soportarse en lo social, ecológico y económico, por lo que solo relacionando adecuadamente estos tres aspectos de la sustentabilidad, es posible proponer proyectos de desarrollo sustentable que busquen "incorporar nuevos elementos académicos hacia una política pública en el campo mineroambiental, requiere confrontar supuestos básicos con los cuales se ha conducido la economía por décadas" (Suárez, 2013, p. 128). Lo que nos muestra que al realizar actividades antrópicas controladas, el territorio no tiene por qué verse afectado, "por las capacidades intrínsecas de restituir su equilibrio original (...) en este sentido, la oposición sociedad/ambiente se aborda desde un lugar que permite la evolución de las actividades tecnológicas" (March, 2005, p. 19).

Otra postura es: el territorio se transforma y organiza, "la naturaleza no parece estar al margen, pues sus recursos son valorados y devorados por el sistema económico" (Shmite, 2008, p. 64), sin embargo, realizar actividades sustentables capaces de insertarse en un mercado cada vez más globalizado hace necesario analizar de manera particular cada territorio, desde un tipo de inserción que permita "sostener y mejorar la calidad de vida de la sociedad actual, respetando sus valores, sus conocimientos, su cultura y, fundamentalmente, sosteniendo la integridad del ambiente natural" (Shmite, 2008,p. 70) por ende el reto de la sustentabilidad subyace en pensar en las sociedades futuras, partiendo de "singularidades locales y construir una racionalidad capaz de integrar sus diferencias, asumiendo su inconmensurabilidad, su relatividad y su incertidumbre... Es resistencia a la hegemonía homogeneizante de la globalización económica y afirmación de la diversidad creativa de la vida, construida desde la heterogénesis cultural-ecológica” (Leff, 2006, p. 271); lo que redundará en la consolidación de una identidad sociocultural, que mantendrá sus distancias frente a los poderes hegemónicos y alivianará las tensiones mercaderistas

frente a la lógica económica que se ha constituido como el más alto grado de racionalidad del ser humano, ignorando a la naturaleza y a la cultura, generando un proceso entrópico insustentable que gobierna la degradación ecológica y la existencia humana (Leff, 2006, p. 269).

\section{Territorio como construcción social}

Se encuentran en esta dimensión, como se puede observar en (Bustos, 2015, p. 128), investigaciones que consideran el territorio como espacio de supervivencia, que permite a los sujetos y las comunidades en general validar su desarrollo cultural, pertinencia e identidad, donde el conocimiento como lo expresan Molina (2010), El-Hani y Mortimer (2007), se construye de manera colectiva, tomando como punto de partida un contexto particular en el cual los individuos interactúan y resaltan la importancia de la cultura en el desarrollo y validación de sus creencias, que son el 
marco de referencia para desarrollar la construcción de signos, que dan significado por la interpretación contextual y que son necesarios para el desarrollo socio-cultural de una comunidad.

Al indagar en el discurso del territorio como una construcción social, se encuentran trabajos en geografía, educación, historia, ciencias políticas, economía, sociología, antropología, entre otras. Lo que permite resaltar de inmediato casi que en todas las posturas las ideas Santos (2000), Fals (2000) y Boisier (2007) quienes con sus investigaciones aportaron al conocimiento del territorio, convirtiéndolo en el gran intermediario entre las diferentes esferas de la sociedad de acuerdo con sus potencialidades y usos específicos. Otro aspecto a tener en cuenta lo presentan entre otros investigadores Fals (2000) y Boiser (2007), para quienes en la medida que el territorio contenga a una comunidad, esta históricamente encontrará en él un referente de su identidad, dado que es allí en donde (Escobar, 2012) se demarcan todas las condiciones de vida y uso de los recursos naturales. Así, la actividad económica estará en mediación constante por los actores y las dinámicas territoriales. En este sentido, el territorio surge como elemento de mediación y de integración con el mundo, que en términos de Quijada (2000) y Sosa (2012) vincula a la dimensión simbólica, las acciones y los pensamientos que dan sentido al espacio y lo convierten en territorio. Por su parte Bondel (2008) propone que antes de abordar el término "territorio", se debe centrar la atención en la manera de ver el espacio; para él, debe ser integral, de tal manera que un espacio condicionado depende del contexto cultural y de las exigencias y explicaciones que este requiera. Como ejemplo, presenta un estudio a las transformaciones territoriales que ha sufrido la comarca andina del paralelo 42, sostiene que estas transformaciones obedecen a dos circunstancias; la primera, centrada en el interés por acompañar intelectualmente los procesos transformadores de una realidad compleja y dinámica. La segunda, tiene relación con el desenvolvimiento incierto de las comarcas andino patagónicas; además, sostiene que estamos actualmente ante "territorios en plena gestión" entendidos como un espacio social con actividad humana, en la que el espacio esta socialmente construido y apropiado, relacionado con "el lugar y el tiempo".

Por su parte Pancho (2007), Guy (2008) y Bondel (2008) coinciden en afirmar que el territorio se construye por las tensiones manifiestas en las diferencias de pensamiento, imaginarios he identidades con el territorio. El ejemplo está presente en la relación que tienen las comunidades indígenas, quienes al referirse a la madre tierra, convergen en afirmar con una visión de desarrollo sustentable ${ }^{11}$

Ella nos devuelve buenas cosechas, ella nos anima, nos regaña y nos apoya (...) Es aquí donde los rituales van tomando significado para recrear esta relación y mantenerla de la mejor manera y son los médicos tradicionales los orientadores y guardianes espirituales de estas prácticas culturales (Pancho, 2007, p. 56).

11 “...los discursos sustentables abren las compuertas de las aguas represadas y de los saberes reprimidos. Buscan arraigar nuevos territorios de vida, decantarse en nuevas racionalidades e incorporarse en nuevas subjetividades" (Leff, 2008, p. 18). 
Contribuyendo a esta misma línea del análisis, en la relación cultura y territorio, están los trabajos de Llanos (2010), Giraldo (2010), Nates (2006), Motta (2006), Ravenet (2002), Giménez (1999), quienes coinciden en afirmar por sus investigaciones, que las comunidades al relacionarse con el contexto dan sentido al espacio habitado, convirtiéndolo en territorio de vida e interacción sociocultural, con caracterización que permiten diferenciarlos y valorizarlos. En el mismo sentido, Álvares y Rendón (2010), reconocen que el desarrollo de una comunidad está en la relación que ella tenga con otras comunidades y con su territorio, por lo que debe existir una competitividad, en el mejor sentido de la palabra, procura alcanzar el desarrollo de la comunidad al aprovechar todo el capital acumulado en el tiempo y resalta los aspectos "físico, humano, tecnológico, institucional, social, cultural, ambiental y natural". Este proceso, en términos de Santos (2000), lleva a interpretar que toda la gama de relaciones que interactúan en el paisaje de un territorio se da en procesos de la abstracción de los lugares y las identidades individuales. Por lo que el paisaje es, y forma parte de lo que Milton Santos denomina "configuración territorial", caracterizada por la función que se le otorga a objetos estáticos y que les permite adquirir su significado de acuerdo con la configuración territorial.

Por su parte, Maris (2008) y Jong (2008) en sus investigaciones coinciden en que el territorio obedece a una construcción social, que en su dinámica contiene muchos matices que se relacionan y que de acuerdo con Bozzano y otros (2012), un territorio basa toda su relación económica y productiva, con las acciones y actividades tradicionales orientadas a un desarrollo que motive el trabajo conjunto de las comunidades, articuladas con la academia, empresarios y políticas estatales en pro de una cooperación y reconocimiento socio-territorial, sin este reconocimiento no van a funcionar las políticas sociales, culturales y educativas.

Por todo lo anterior, el territorio emerge como consecuencia de la construcción de significados, convergencia de expectativas, sueños y relaciones intra e interculturales, donde los códigos propios de estas relaciones se relacionan con aspectos jurídicos, políticos, económicos, sociales, culturales y ambientales.

No se puede continuar sin antes entablar un diálogo con lo que hemos denominado "Un territorio socialmente construido y amenazado"; referimos aquí, a todas las acciones violentas ejercidas por actores externos al territorio, que han afectado y afectan los territorios construidos por las comunidades; transformándolos y condicionándolos a su voluntad, lo que ha conducido a procesos de desterritorialización, como ha sucedido históricamente en Colombia, y queda expuesto en la investigación de CEPAL (2012), cuando aseguran que:

la no sustentabilidad del medio ambiente, la contaminación, el cambio climático y la depredación de los recursos ubicados en tierras ancestrales de los pueblos y comunidades indígenas y afrodescendientes ha significado que estas poblaciones no puedan continuar utilizando sus recursos naturales fundamentalmente el agua-, mantener su relación con la tierra y el consumo 
balanceado de sus frutos, lo que ha generado altos niveles de pobreza, emigración, desplazamiento y enfermedades (Cepal, 2012, p. 30).

En el mismo sentido, como lo presenta (Bustos 2015), al abordar los trabajos de Nates (2006) y Burbano (2014) resalta que en sus investigaciones han abordado el problema del desplazamiento forzado en Colombia; como lo afirma Nates (2006) al referir a la apropiación material y simbólica del espacio, hace referencia a un proceso de construcción social y caracterización de espacios de conflicto.

Para ella, las apropiaciones del espacio desde lo material y simbólico, evidencia el proceso de construcción del territorio, en este sentido, las regularidades y significados que se ciernen en este proceso de formas de organización y de creaciones culturales, permiten la construcción de un sólido vínculo con el territorio. Si la idea anterior la trasladamos al conflicto colombiano, en donde la muerte por violencia es evidente, es precisamente en esta dinámica que surge la categoría de 'territorio abandonado' (Bustos, 2014, p. 90).

Lo anterior, sumado a la idea de que "los paramilitares, que surgen como una expresión de grandes ganaderos y narcotraficantes para detener el avance de la guerrilla, se proponen limpiar de guerrilleros y simpatizantes los territorios dominados por las fuerzas insurgentes" (Castillo, 2006, p. 584). Invita a pensar en aquellos territorios ancestrales que han sido objeto de invasión y robo de sus recursos naturales, no solo por fuerzas armadas como guerrilla y paramilitares, sino por otros actores como narcotraficantes y gobernantes corruptos, para quienes no interesa la diversidad cultural.

No obstante, las comunidades se han resistido a estos avatares y esta resistencia los conduce a la idea de no abandonar el territorio

por lo que actores externos y sin escrúpulos han ejercido presión sobre líderes indígenas; varios han sido señalados y asesinados por sus actividades, generando desplazamientos de población. Asimismo, hay desconocimiento de la autonomía indígena y de sus derechos fundamentales, al controlar tanto el acceso a sus territorios como a sus alimentos (Ulloa, 2010, p. 84).

Sin embargo, la resistencia ante estos avatares permite no perder la identidad y la motivación social. Por tal razón, las luchas de las comunidades y la cooperación del Estado deben permitir la protección, el reconocimiento de la tenencia del territorio a las comunidades y el apoyo institucional a la producción y el desarrollo. Sin estas acciones no van a funcionar, entre otras, las políticas educativas, sociales y culturales. Por este motivo, es primordial la validación y el respeto a la diversidad cultural, arraigada en un territorio donde el "Territorio y territorialidad es pues la interacción entre ecosistemas y cultura, que incluye formas organizativas propias, tecnología disponible, economía y producción simbólica" (Motta, 2006, p. 12).

Como lo expresa (Bustos, 2015, p. 136) al citar el trabajo de Giraldo (2010); para los pueblos indígenas serranos el territorio no solo es un espacio de corteza 
terrestre, por el contrario es allí donde se hace posible el desarrollo y supervivencia de una comunidad. La sociedad influye en el territorio, lo transforma, lo modifica, lo utiliza y lo amenaza. En tal sentido, el hombre convierte a través de los procesos de territorialización al espacio natural, en espacio humanizado. Por lo tanto, la desterritorialización está relacionada directamente con la angustia e inestabilidad de los sujetos, que en ausencia del espacio habitado anhela volver a su territorio, reterritorializarlo lo que hace que las actividades de las comunidades sean dinámicas, para el caso de las comunidades indígenas, dada la importancia que estas le dan al territorio inmaterial como elemento articulador social desde dos aspectos;

..., por un lado procesos de reconocimiento parcial e instrumental, y por otro una gobernabilidad parcial y limitada sobre sus territorios. Estos procesos exigen permanentemente a los pueblos indígenas, la generación de estrategias de construcción de alianzas, reconsideración de procesos externos y reconfiguración de procesos internos para establecer negociaciones y relaciones con otros actores sociales (Ulloa, 2010, p. 78).

Lo que ocurre es una visión de territorio, como espacio terrestre socialmente construido, por lo tanto, va mucho más allá de lo que teóricamente se ha entendido, en donde no solo es concebido como continente físico, por el contrario, al referirnos a socialmente construido no podemos desligarlo de su significado histórico-cultural, por lo que:

El territorio es un lugar de variada escala -micro, meso, macro- donde actores -públicos, privados, ciudadanos, otros- ponen en marcha procesos complejos de interacción -complementaria, contradictoria, conflictiva, cooperativa, solidariaentre sistemas de acciones y sistemas de objetos, constituidos éstos por un sinnúmero de técnicas -híbridos naturales y artificiales-e identificables según instancias de un proceso de organización territorial en particulares acontecimientos -en tiempo-espacio- y con diversos grados de inserción en la relación local-mesoglobal. El territorio se redefine siempre (Bozzano, 2009, p. 146).

\section{Territorio como espacio físico}

Basado en la investigación sobre concepciones de territorio que realizaron Bozzano, Gaston y D'Amico (2008); quienes (durante una década de 1998 a 2008) encontraron después de analizar las nociones y prenociones referidas al concepto de territorio que tienen 540 universitarios que pertenecen a distintas disciplinas científicas, concluyeron que la mitad de los casos $(53,7 \%)$ se trata de un concepto vinculado a la definición de territorio como un espacio físico que es escenario y soporte de las actividades humanas. Esto hace que sea importante indagar en investigaciones que se interesen en las concepciones de la transformación de los paisajes producidos por diferentes eventos naturales y otros provocados por los seres humanos; en particular, aquellas representaciones mediadas por tecnologías propias de las ingenierías, cuyo objeto-estudio son las ciencias de la tierra. Por 
tanto, se incluyen en esta dimensión, aquellos trabajos e investigaciones en los que no son de interés los debates desde la filosofía, sociología, antropología y afines. Por el contrario, permanece vigente "la imagen que dio origen a su nombre: geografein= descripción de la tierra, por extensión, del espacio terrestre; aún perduran expresiones del tipo (la geografía estudia montañas, ríos, actividades económicas, lugares, regiones)" (Bozzano, 2009, p. 45).

Al indagar en estudios sobre el territorio desde la concepción de fronteras, Ulloa (2010) presenta, como para algunos casos las fronteras se vuelven porosas cuando para el caso colombiano, por los avatares del enfrentamiento armado y la presencia de cultivos ilícitos, las fronteras se reconfiguran y se entremezclan con las disputas territoriales que demandan las comunidades ancestrales por la usurpación de su territorio "como es el caso del Puerto Brisa, en donde en el transcurso de 10 años aproximadamente, se pasó de un reconocimiento a una negación del territorio ancestral indígena" (Ulloa, 2010, p. 90), aquí para los actores ajenos al territorio indígena son de interés los pastos para la ganadería y las minas para su explotación. Los sentimientos y relaciones de las comunidades por su territorio pasan a un segundo plano. Por su parte, Meza (2006) al estudiar el Bajo Atrato colombiano argumenta, que las fronteras que delimitan los territorios han permanecido, el paisaje define sus características y fronteras. En este sentido, muestra cómo en el Bajo Atrato existen tensiones por el dominio territorial. Por un lado están los afrodescendientes que habitan las riberas de los ríos, para quienes los ríos y los bosques son un factor preponderante para realizar un aprovechamiento de los recursos naturales. En oposición a ellos encontramos a los llamados por los habitantes del Choco como Chilapos y los Paisas, quienes tienden a invadir las zonas selváticas para aumentar la frontera de paisaje sabanero propicio para la ganadería y el cultivo a gran escala terrestre con características particulares que la diferencia de otras.

En relación con las diferentes esferas del conocimiento en las que se evidencia un estudio del territorio relacionado con el análisis de los suelos y sus propiedades, hace necesario tener en cuenta trabajos relacionados, entre otros, con los Sistemas de Información Geográfica, Cartografía, Geodesia, Topografía, Geofísica y aspectos tecnológicos que median estas representaciones sobre el territorio y que se constituyen en temas de central importancia en programas de pregrado en donde se desempeñan los profesionales de ciencias de la tierra.

En el trabajo realizado por Álvarez y otros (1989), el territorio está ligado al concepto de poder, de manera que los límites territoriales son definidos como el espacio físico, donde los sistemas de información geográfica (SIG) surgen como herramienta que permite el análisis y representación de mapas que son instrumentos y sirven para georreferenciar. Estos estudios aportan información en torno a la gestión y análisis de la información espacial, planificación ambiental, ordenamiento territorial, aquí se tiene en cuenta la distribución de suelos según su perfil, características del terreno, escenarios que se deben tener en cuenta según intenciones de los actores 
y preferencia de exclusión (políticas del Gobierno) que permiten determinar los factores que intervienen en el relieve, en los que se tiene en cuenta la valoración ambiental y funcional del territorio.

Con los trabajos de Álvarez y otros (1989), el territorio como espacio físico, se refiere a la superficie terrestre que provee de bienes y alimentos a las comunidades, por lo que realizar todos los estudios de cartografía ${ }^{12,}$ percepción remota y sistemas de información geográfica, son de importancia en el ordenamiento y planificación territorial.

Con el trabajo de Montalvo y Silva (2009) se reconoce el territorio como un espacio físico, aquí el referente de representación es el mar y su litoral, en particular, la costa Caribe colombiana. Los autores muestran, en el desarrollo del documento, la existencia de las diferentes riquezas naturales. Por su parte, son numerosos los trabajos en ciencias de la tierra donde prevalece el estudio del espacio físico, lejos de las dinámicas socioculturales que en él se dan, ejemplo de ello son los trabajos de Jiménez (2009) y López, Nieto y Arias (2010), para quienes el territorio es visto como una delimitación del espacio físico, lo que implica centrar esfuerzos en estudiar problemas de movilidad, y de ocupación física del territorio, relación dinámica entre el espacio urbano y el espacio rural en relación con la posible densidad poblacional y las unidades habitacionales, en una época en la que la desterritorialización de la población rural implica el crecimiento de la población urbana. Por lo que, observar con mayor claridad las características físicas de un territorio, al estudiar con mayor detenimiento, el paisaje, la distribución urbana y rural, los suelos, la biodiversidad, bosques, matorrales y pastos, líneas eléctricas, zonificación, riesgos geológicos, densidad de la población, vías, entre otros.

\section{Conclusiones}

La relación de los trabajos presentados evidencian la prolífera interpretación que se le puede dar al territorio y su importancia en la formación de futuros profesionales de las ciencias de la tierra, por ello Molina y Martínez (2009), como actor principal en la formación pone en juego sus concepciones territoriales, relacionadas con su actividad profesional y acción docente, que debe estar en consonancia con las posturas de Longás y otros (2008), Arango y Pulgarín (2010), Champollion (2011), Castro (2012), quienes coinciden en que los impactos territoriales que sufren las comunidades afectan el progreso educativo de los estudiantes y la misma organización de los centros de estudio en cualquiera de los niveles de la educación.

12 La Cartografía, es entendida como la ciencia capaz de ofrecer una representación gráfica lo más fiable posible del territorio; mostrar de forma reducida, sintética y esquemática una realidad compleja e incluso a veces difícilmente abarcable, y a través de ello obtener una mejor comprensión de los fenómenos de la Tierra, su localización, tamaño y distribución encarga entre otras cosas de la elaboración del mapa oficial de Colombia así como diferentes mapas topográficos, también es la encargada de establecer la red geodésica nacional. 
Por lo que realizar trabajos que estudien las dinámicas y relaciones del territorio con el entorno, lleva a la comunidad a reflexionar y abordar nuevos paradigmas que "rescaten los valores de la comunidad educativa para abrirse a su entorno y aportar para el capital social de las nuevas generaciones", para lograrlo, es indispensable reconocer el papel de la diversidad cultural y así comprender las diferentes relaciones de las comunidades con su entorno. Por lo anterior, y de acuerdo con la postura de Steinberg, Centrángolo y Gatto (2011) (quienes después de realizar una investigación sobre las implicaciones que tienen 26 contextos territoriales diferenciados en la educación), las diferencias de contexto generan diversos imaginarios territoriales. Por lo que repensar en propuestas académicas, según los variados contextos socio culturales, implica reconocer, respetar y valorar que en el país coexisten diferentes actores con concepciones disímiles, acordes con las necesidades propias de su contesto territorial o del contexto territorial del que han sido desplazados y que llegan a aumentar los cordones de miseria, como es el caso de Bogotá (Burbano, 2014) a donde llegan la mayor cantidad de desplazados, ubicándose en las localidades periféricas de la ciudad.

\section{Referencias}

Álvarez, J., Yanguas, M., Rodríguez, M., y Gómez, S. (1989). Condicionantes geofísicos de los usos del territorio en un valle de la montaña Cantábrica. Options Méditerranéennes Série Séminaires, 03, 283-288. Recuperado de http:// om.ciheam.org/om/pdf/a03/CI000552.pdf

Álvares, R., y Rendon, J. (2010). El territorio como factor del desarrollo. Semestre Económico, 13(27), 39-62, Medellín, Colombia.

Arango, J., y Pulgarín, M. (2010). Los estudios del territorio y la educación geográfica. Una mirada desde el PUI-NOR, Medellín-Colombia. Uni-pluri/versidad, 10(3), 1-10. Versión Digital Facultad de Educación, Universidad de Antioquia. Medellín, Colombia. Recuperado de http://aprendeenlinea.udea.edu.co/revistas/index.php/ unip/article/viewFile/9585/8825

Boisier, S. (2007), Imágenes en el espejo. Aportes a la discusión sobre crecimiento $y$ desarrollo territorial. Toluca, Universidad Autónoma del Estado de México.

Bondel, S. (2008). Transformaciones territoriales y análisis geográfico en ámbitos patagónicos de montaña. La comarca Andina del paralelo 42. (Tesis doctoral). Universidad de La Plata. Argentina. Recuperada de http://www.memoria.fahce. unlp.edu.ar/tesis/te.281/te.281.pdf

Bozzano, H. (2009). Territorios reales, territorios pensados, territorios posibles. Aportes para una teoría territorial del ambiente. Buenos Aires: Editorial Espacio. 
Bozzano, Gaston, y D'Amico. (2008). Territorios posibles procesos, lugares y actores. Buenos Aires: Lumiere.

Bozzano, H., Escudero, L., Oggero, C., Núñez, R., y García, V. (2012). Observatorio OIDTe Lavalleja de inteligencia y desarrollo territorial, Uruguay 2010-2012. En Inteligencia territorial. Teoría, métodos e iniciativas en Europa y América Latina. Bozzano (Ed.). Universidad Nacional de La Plata. Argentina.

Burbano, D. (2014). Lectura del desplazamiento forzoso por la violencia en Colombia, desde el territorio y las condiciones habitacionales. El caso de estudio de Bogotá. (Tesis doctoral). Escuela Técnica Superior de Arquitectura de Madrid.

Bustos, E. (2014). La importancia de la relación cultura, territorio y enseñanza de las ciencias (Cap. $4^{\circ}$.). En Enseñanza de las ciencias y cultura: Múltiples aproximaciones. Serie grupos $\mathrm{N}^{\circ}$ 5. Fondo de Publicaciones. Universidad Distrital Francisco José de Caldas. Bogotá, Colombia.

Bustos, E. (2015). Contribuciones metodológicas para el estudio de las concepciones de territorio de profesionales de ciencias de la tierra. Capítulo sexto. En Educación en ciencias: Experiencias investigativas en el contexto de la didáctica, la historia, la filosofía y la cultura. Fondo de Publicaciones. Universidad Distrital Francisco José de Caldas. Bogotá, Colombia.

Castro, M. (2012). Política, educación y territorio en Chile (1950 - 2010). De las acciones colectivas a las acciones del mercado. Educación y Educadores, 15(1), 97-114. Recuperado de http://www.scielo.org.co/pdf/eded/v15n1/v15n1a07

Castillo, J. (2006). El Estado-nación pluriétnico y multicultural colombiano: la lucha por el territorio en la reimaginación de la nación y la reivindicación de la identidad étnica de negros e indígenas. (Tesis doctoral). Universidad Complutense de Madrid. Recuperado de http://biblioteca.ucm.es/tesis/cps/ucm-t28946.pdf

CEPAL. (2005). Pueblos indígenas y afrodescendientes de América Latina y el Caribe. Publicación de las Naciones Unidas. Santiago de Chile. Recuperado de http://www.cepal.org/mujer/noticias/noticias/5/27905/pueblosindigenas_afro.pdf

CEPAL. (2012). Atlas sociodemográfico de los pueblos indígenas y afrodescendientes en Colombia. Publicación de las Naciones Unidas. Santiago de Chile. Recuperado de http://repositorio.cepal.org/handle/11362/1264

Champollion, P. (2011). El impacto del territorio en la educación. El caso de la escuela rural en Francia. Profesorado. Revista de currículum y formación del profesorado, 15(2), 53-69. Recuperado de http://www.ugr.es/ recfpro/rev152ART4.pdf

ConstituciónPolíticadeColombia.(1991)Recuperadodehttp://www.contraloriapereira.gov. co/files.php?fid=24\&fcid=2\&PHPSESSID $=0$ af317a9c52c6247711881ae5e $8 \mathrm{~d} 5551$ 
Coronado, S. (2006). El territorio: derecho fundamental de las comunidades afrodescendientes en Colombia. Controversia, 187, 47-81. Recuperado de http://bibliotecavirtual.clacso.org.ar/Colombia/cinep/20100920091337/ art03territorioControversia187.pdf

El-Hani, C., \& Mortimer, E. (2007). Multicultural education, pragmatism, and the goals of science teaching. Cult Stud of Sci Educ, 2, 657-685. Recuperado de http:// blogs.springer.com/csse/wp-content/uploads/2008/03/Feature\%20Focus\%20 Forum_Vol2(3).pdf

Escobar, I. (2012). Observatorio en territorio educador: crencias y prácticas investigativas y pedagógicas, para una didáctica del medio ambiente. En Investigación y educación ambiental. Apuestas investigativas pertinentes a los campos de reflexión e intervención en educación ambiental. Torres, M. (ed). Edición Especial. Impresores Ltda. Bogotá, D.C., Colombia.

Fals, O. (2000). El territorio como construcción social. Revista Foro, 38, 45-51

Geertz, C. (1992). La interpretación de las culturas. Gedisa, Barcelona.

Giménez, G. (1999). Territorio, Cultura e Identidades: la región socio-cultural. Estudios sobre las culturas contemporáneas. Época II. (9), 25-57.

Giraldo, N. (2010) Camino en espiral. Territorio sagrado y autoridades tradicionales en la comunidad indígena Iku de la Sierra Nevada de Santa Marta, Colombia. Pueblos y Frontera, 6, 9. Recuperado de http://www.pueblosyfronteras.unam.mx/a10n9/ art_07.html

Guy, D. (2008). Une géographie sociale entre représentations et action. Montagnes méditerranéennes et developpement territorial. $H A L I d, 23,1-8$. Recuperado de $\mathrm{http} /$ halshs.archives-ouvertes.fr/halshs-00281573/document

Irastorza, P. (2006). Integración de la ecología del paisaje en la planificación territorial. Aplicación a la comunidad de Madrid. (Tesis doctoral). Universidad Politécnica de Madrid. Recuperado de http://oa.upm.es/468/1/PEDRO_ IRASTORZA_VACA.pdf

Jiménez, F. (2009). Hacia la consolidación del territorio urbano del distrito capital de Bogotá. Revista de ingeniería, 29, 96-99.

Jong, G. (2008). Análisis regional, estructuras agrarias y estrategias de desarrollo regional en la fruticultura del Alto Valle de la cuenca del Río Negro. (Tesis doctoral). Universidad Nacional de La Plata, Facultad de Humanidades y Ciencias de la Educación. Recuperado de http://www.memoria.fahce.unlp.edu.ar/tesis/ te.272/te.272.pdf

Leff, E. (2008). Discursos sustentables. México: Editores Siglo XXI. 
Leff, E. (2006). La geopolítica de la biodiversidad y el desarrollo sustentable. Economización del mundo, racionalidad ambiental y reapropiación social de la naturaleza. Observatorio Social de América Latina, 17 (VI), 263-273. Recuperado de http://bibliotecavirtual.clacso.org.ar/clacso/osal/20110313071126/37Leff.pdf

Longás, J., Civís, M., Riera, J., Fontanet, A., Longás, E., y Tomás, A. (2008). Escuela, educación y territorio. La organización en red local como estructura innovadora de atención a las necesidades socioeducativas de una comunidad. Sips-revista Interuniversitaria de Pedagogía Social, 155, 137-151. Recuperado de http://www. redalyc.org/articulo.oa?id=135012674011

López, F., Nieto, D., y Arias, C. (2010). Relaciones entre el concepto de movilidad y la ocupaciónterritorialdeMedellín.RevistaEIA, 13,23-37. Recuperadodehttp://www. scielo.org.co/scielo.php?script $=$ sci_arttext\&pid=S1794-12372010000100003

Llanos, L. (2010). El concepto del territorio y la investigación en las ciencias sociales. Agricultura, Sociedad y Desarrollo, 7 (3), 213-220. Recuperado de http://www. redalyc.org/articulo.oa? $\mathrm{id}=360533086001$

Mançano, B. (2008). Sobre la tipología de los territorios. Recuperado de http:// web.ua.es/es/giecryal/documentos/documentos839/docs/bernardo-tipologia-deterritorios-espanol.pdf

March, J. (2005). Nuevos fundamentos de racionalidad ambiental a partir del análisis epistemológico de la evaluación del impacto ambiental. Cinta de Moebio, 24, $265-$ 287. Universidad de Chile. Recuperado de http://www.investigacionesgeograficas. uchile.cl/index.php/CDM/article/view/26074/27379

Maris, S. (2008). Territorio y sustentabilidad. El "caldenal" en la lógica actual del capitalismo. Revista Iberoamericana de Economía Ecológica, (9), 61-73. Recuperado de http://www.redibec.org/IVO/rev9_05.pdf

Meza, C. (2006). Territorios de frontera: Embate y resistencia en la cuenca del río Cacarica. Universitas humanística, (62), 385-429. Recuperado de http://www. javeriana.edu.co/Facultades/C_Sociales/universitas/62/meza.pdf

Ministerio de Ambiente y Desarrollo Sostenible. (2012). Educación ambiental. Política nacional. Torrez, M. (Ed.). Edición Especial. Bogotá, D.C., Colombia: Impresores Ltda.

Molina, A., y Martínez, C. (2009). Concepciones de los profesores: perspectivas para su estudio en contextos culturales diversos. Enseñanza de las Ciencias, 3180-3185. Número Extra. VIII Congreso Internacional sobre Investigación en Didáctica de las Ciencias, Barcelona. Recuperado de http://ensciencias.uab.es/ congreso09/numeroextra/art-3180-3185.pdf 
Molina, A. (2010). Una relación urgente: Enseñanza de las ciencias y contexto cultural. EDUCyT, 1(1), 1-12.

Montalvo, A., y Silva, F. (2009). El mar ¿territorio de quién? Algunos elementos para una propuesta de una antropología del litoral. Universitas Humanística, (68), 247265. Recuperado de http://www.redalyc.org/articulo.oa?id=79118995013

Moreno, A., y Ramírez, J. (2006). Pierre Bourdieu introducción elemental. Bogotá: Panamericana.

Moreno, M. (2010). Génesis, evolución y tendencias del paradigma del desarrollo sostenible. México: Editorial Miguel Angel Porrua.

Motta, N. (2006). Territorios e identidades, centro de estudios de género, mujer y sociedad. Historia y Espacio, (26), 91-109.

Nates, B., y Raymond, S. (2006). Cartografía semiótica para la comprensión de territorios de conflicto. Estudios Políticos, (29), 99-120.

Osorio, L. (2001). Planes de acción ambiental local y terrritorio. En Vásquez, E. (Ed.). Espacio y territorios: Razón, pasión e imaginarios. Bogotá, Colombia: Universidad Nacional de Colombia. Unilibros.

Otero, A. (2006). La importancia de la visión de territorio para la construcción de desarrollo competitivo de los destinos turísticos. Cuadernos de Turismo, (19), 91104. Recuperado de http://www.redalyc.org/pdf/398/39801905.pdf

Pancho, A. (2007). Participación de las mujeres Nasa en los procesos de autonomía territorial y educación propia en el Cauca, Colombia. En Ulloa, A. (ed.). Mujeres indigenas, territorialidad y biodiversidad en el contexto latinoamericano. Universidad Nacional de Colombia, Bogotá.

Quijada, M. (2000). Indígenas: Violencia, tierras y ciudadanía. En Quijada, M. et all. Homogeneidad y nación. Con un estudio de caso: Argentina, siglos XIX y XX. Madrid: Csic.

Quijada, M. (2006). La caja de pandora. El sujeto político indígena en la construcción del orden liberal. Historia Contemporánea, (33), 605-637. Recuperado de file://I Users/edierhernan/Downloads/4185-14725-1-PB.pdf

Ravenet, M. (2002). Espacio y territorio en los estudios sociológicos en Cuba. (Tesis doctoral). Universidad de La Habana. Recuperado de www.yumpu. com/es/document/view/14208156/tituloespacio-y-territorio-en-los-estudiossociologicos-en-cuba

Sánchez, G. et all. (2009). El despojo de tierras y territorios. Aproximación conceptual. Área de Memoria Histórica Comisión Nacional de Reparación y Reconciliación. Línea de Investigación Tierra y Conflicto. Recuperado de www. memoriahistorica-cnrr.org.co 
Santos, M. (2000). La naturaleza del espacio, técnica y tiempo. Razón y emoción. Barcelona. España: Ariel.

Sauvé, L. (2003). Perspectivas curriculares para la formación de formadores en educación ambiental. México: Universidad Autónoma de San Luis Potosí.

Semper, F. (2006). Los derechos de los pueblos indígenas de Colombia en la jurisprudencia de la Corte Constitucional. Anuario de Derecho Constitucional Latinoamericano, 761-778. Recuperado de www.zotero.org/spartakku/items/ itemKey/3JPV7FUQ

Shmite, S. M. (2008). Territorio y sustentabilidad: El "cardenal" en la lógica actual del capitalismo. Revista Iberoamericana de Economía Ecológica (REVIBEC), (9), 61-73.

Sosa, M. (2012) ¿Cómo entender el território? Guatemala: Editorial Cara Parens.

Steinberg, C., Centrángolo, O., y Gatto, F. (2011). Desigualdades territoriales en la Argentina. Insumos para el planeamiento estratégico del sector educativo. Recuperado de http://repositorio.cepal.org/handle/11362/3853

Suárez, A. (2013). La minería colonial del siglo XXI. No todo lo que brilla es oro. Bogotá, D.C.: Ediciones Aurora.

Ulloa, A. (2010). Reconfiguraciones conceptuales, políticas y territoriales en las demandas de autonomía de los pueblos indígenas en Colombia. Tabula Rasa, (13), 73-92. Recuperado de http://www.revistatabularasa.org/numero-13/03Ulloa.pdf 